\title{
The association of weight gain with nonalcoholic fatty liver disease and fibrosis detected by FibroScan in the United States
}

\author{
Karn Wijarnpreechaa ${ }^{a}$ Elizabeth S. Aby ${ }^{b}$, Aijaz Ahmed*, Donghee Kim ${ }^{\text {c* }}$ \\ University of Michigan, Ann Arbor, MI; University of Minnesota, Minneapolis, MN; Stanford University School of \\ Medicine, Stanford, CA, USA
}

\section{Abstract}

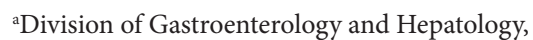

University of Michigan, Ann Arbor, MI (Karn Wijarnpreecha);

bDepartment of Medicine, Division of Gastroenterology and

Hepatology, University of Minnesota, Minneapolis, MN

(Elizabeth S. Aby); ' Division of Gastroenterology and

Hepatology, Stanford University School of

Medicine, Stanford, CA (Aijaz Ahmed, Donghee Kim), USA

${ }^{\star}$ Joint senior authors

Conflict of Interest: Karn Wijarnpreecha received funding from the AASLD Advanced/Transplant Hepatology Award. Other coauthors have no conflict of interest

Correspondence to: Donghee Kim, MD, PhD, Division of Gastroenterology and Hepatology, Stanford University School of Medicine, 300 Pasteur Drive, Stanford, CA 94304, USA, e-mail: messmd@chol.com,dhkimmd@stanford.edu

Received 19 October 2021; accepted 23 December 2021; published online 15 February 2022

DOI: https://doi.org/10.20524/aog.2022.0687

\section{Introduction}

Nonalcoholic fatty liver disease (NAFLD) is a growing healthcare burden worldwide, with an estimated global prevalence of 25\% [1]. NAFLD comprises a broad spectrum of diseases, from nonalcoholic fatty liver to nonalcoholic steatohepatitis (NASH), significant fibrosis and end-stage liver disease, such as cirrhosis and hepatocellular carcinoma. Patients with NAFLD and fibrosis have a significantly increased risk of cardiovascular disease and liver-related mortality $[2,3]$. Hospitalization rates and the economic burden from NAFLD have increased compared to other etiologies of liver diseases in the United States (US) [4,5]. It is estimated that NAFLD is the most common cause of the rapidly increasing liver-related mortality worldwide [6].

Obesity has been identified as a risk factor in the pathogenesis of NAFLD and progression to NASH and fibrosis [7]. From 50-75\% of obese individuals have NAFLD [7]. 
Obesity and weight gain were associated with the progression of liver fibrosis, defined by a noninvasive marker, in an Asian population with NAFLD [8]. It has been shown that fibrosis stage predicts severe liver disease and mortality in patients with NAFLD [9]. Although liver biopsy remains the gold standard for histological evaluation, transient elastography is a reliable noninvasive test to measure and monitor liver fibrosis, as well as to assess hepatic steatosis measured by the controlled attenuation parameter (CAP) [10]. A recent meta-analysis reported an association between weight loss interventions and improvement in NASH, but not in fibrosis [11]. Several studies have reported an association between weight gain and ultrasonography-diagnosed NAFLD, as well as fibrosis by noninvasive markers, in Asian control groups [8,12]. However, no studies have evaluated the impact of weight gain over 10 years on the risk of NAFLD as well as NAFLD-associated significant fibrosis, as measured by transient elastography, in the US population. Because of the lack of an approved pharmacologic treatment for NAFLD or fibrosis, there is a need to better understand modifiable risk factors for NAFLD as well as NAFLD-associated fibrosis to reduce the disease burden and associated healthcare costs. Therefore, we aimed to investigate the association between weight gain in the short- and longterm and NAFLD, as well as NAFLD-associated significant fibrosis measured by transient elastography, in the general US population.

\section{Materials and methods}

\section{Study population}

We analyzed data from the contemporary National Health and Nutrition Examination Survey (NHANES) 2017-2018. The NHANES dataset includes anthropometric measures as well as comprehensive questionnaires covering sociodemographic and lifestyle information, including weight history and detailed laboratory tests [13]. Ten-year data concerning weight were available for adults over 36 years; therefore, a total of 3843 participants were identified with a complete weight history. Five hundred forty-nine participants were excluded because of ineligible transient elastography or an incomplete examination. In addition, participants with viral hepatitis (presence of the hepatitis B surface antigen and/or hepatitis C virus RNA; $n=62$ ), exposure to steatogenic medications (such as amiodarone, corticosteroid, methotrexate, tamoxifen and valproate) for more than 6 months $(n=69)$, significant alcohol consumption, defined as $>30 \mathrm{~g} /$ day in men and $>20 \mathrm{~g} /$ day in women [1], $(n=83)$, and incomplete waist circumference data $(n=114)$ were excluded. We also excluded participants with a medical history of fatty liver disease $(n=79)$, medical history of other liver diseases (viral hepatitis, autoimmune hepatitis, primary biliary cholangitis, primary sclerosing cholangitis, $\alpha-1$ antitrypsin deficiency, hemochromatosis, Wilson's disease, alcohol related liver disease, liver cancer, liver fibrosis; $n=32$ ), and participants with HIV $(n=6)$. Finally, a total of 2849 participants were included in this analysis (Fig. 1).

\section{Definition of NAFLD and significant fibrosis}

We used previously proposed methods for the definition of NAFLD and significant fibrosis $[14,15]$. The FibroScan model 502 V2 Touch (Echosens, Waltham, MA) was equipped with medium or extra-large probes, which were used to estimate the CAP score and liver stiffness. Elastography results were considered as invalid and subsequently excluded if any of the following were present: $<10$ complete stiffness measurements out of up to 30 attempts, interquartile range/median ratio $>30 \%$, or $<3$ h of fasting before the examination $[14,16,17]$. We included individuals with serum aminotransferase $<5$ times the upper limit of the normal range. The interobserver reliability was 0.94 for CAP and 0.86 for liver stiffness $[14,16]$. We defined suspected NAFLD $(\geq \mathrm{S} 1)$ as CAP scores $\geq 263 \mathrm{~dB} / \mathrm{m}$ (cutoff of sensitivity fixed at 90\%) [14,18]. For the sensitivity analysis, we defined CAP scores $\geq 285 \mathrm{~dB} / \mathrm{m}$ (cutoff optimizing sensitivity and specificity) as suspected NAFLD [14,18]. While most studies assign a cutoff of $>250 \mathrm{~dB} / \mathrm{m}$ for significant steatosis ( $>33 \%$ of hepatic steatosis), a prior study suggested a cutoff of $288 \mathrm{~dB} / \mathrm{m}$ to detect $>5 \%$ hepatic steatosis [19]. It should be noted that the CAP score can be affected by several factors, such as diabetes, body mass index, and NAFLD [19]. Liver stiffness of $\geq 8 \mathrm{kPa}(\geq \mathrm{F} 2)[10,19,20]$. was used to define significant fibrosis in the cohort and among individuals with NAFLD.

\section{Definition of weight changes}

Weight changes over 10 years were calculated as the difference between present weight and weight 10 years before. Likewise, weight changes over a year were calculated as the difference between present weight and weight 1 year before. Weight changes were categorized into 4 weighted quartiles for 1 and 10 years, respectively. Weight changes over 10 years were categorized as follows: quartile $1,<-7$ pounds $(-3.18 \mathrm{~kg})$; quartile $2,-7$ pounds $(-3.18 \mathrm{~kg})$ to 6 pounds $(2.72 \mathrm{~kg})$; quartile 3,6 pounds $(2.72 \mathrm{~kg})$ to 20 pounds $(9.07 \mathrm{~kg})$; and quartile $4, \geq 20$ pounds $(9.07 \mathrm{~kg})$. For the weight changes over a year, we categorized quartiles as follows: quartile $1,<-5$ pounds $(-2.27 \mathrm{~kg})$; quartile $2,-5$ pounds $(-2.27 \mathrm{~kg})$ to 0 pounds; quartile $3,0-4$ pounds $(1.81 \mathrm{~kg})$; and quartile $4, \geq 4$ pounds $(1.81 \mathrm{~kg})$. We used quartile 2 (weight-stable group) as the reference group. We categorized quartile 3 and quartile 4 as the weightgain group and quartile 1 as the weight-loss group.

\section{Statistical analysis}

In view of the complex sampling design employed by the NHANES, we used appropriate sample weights to reconstruct representative data of the US population. According to quartiles for weight changes, baseline characteristics were compared using the $\chi^{2}$-test for categorical variables or linear regression for continuous variables. Data are shown as the weighted mean \pm standard error or weighted frequency with $95 \%$ confidence 
NHANES 2017-2018

Adults $\geq 36$ years and available weight history questionnaire $(n=3,843)$

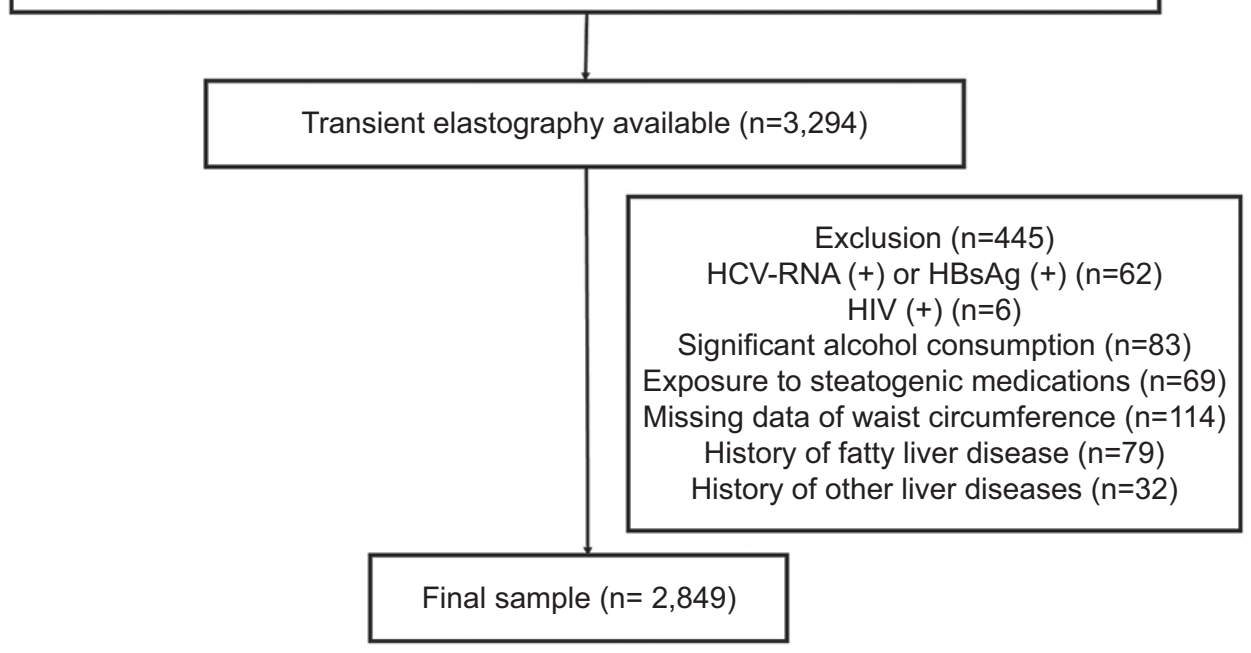

Figure 1 Flow chart showing cohort selection

intervals (CI), as appropriate. We used multivariate logistic regression analyses to determine the independent association between weight changes, NAFLD and NAFLD-associated fibrosis, after adjusting for multiple demographic and metabolic confounders, including age, sex, race/ethnicity, education level, marital status, smoking status, hypertension, diabetes, total cholesterol, high-density lipoprotein-cholesterol, physical activity, and weight circumference (model 2). We performed analyses using STATA 15.1 (StataCorp., College Station, Texas, USA).

\section{Results}

\section{Weight changes over 10 years and NAFLD/significant fibrosis}

As shown in Table 1, individuals in the weight-gain groups (quartiles 3 and 4) tended to be younger, female, Hispanic, non-Hispanic Black, current smokers, and more likely to have hypertension and diabetes compared to individuals in the weight-stable group. As expected, individuals in the weightgain group had a higher body mass index, waist circumference, alanine aminotransferase, and gamma-glutamyl transferase than individuals in the weight-stable group. The prevalence of NAFLD (CAP scores of $\geq 263 \mathrm{~dB} / \mathrm{m}$ ) in the weight-gain group (68.8\%,95\%CI 61.5-75.3 for quartile 4) was higher compared to the weight-stable group (44.4\%, 95\%CI 38.4-50.6 for quartile 2). Using another definition for NAFLD (CAP scores $\geq 285 \mathrm{~dB} / \mathrm{m}$ ), similar trends were noted (Table 1 ). The prevalence of NAFLD in the weight-loss group (quartile 1) was slightly higher than or comparable with that in the weight-stable group.

Table 2 shows univariate and multivariate analyses regarding the association between weight changes over
10 years and NAFLD. When NAFLD was defined as a CAP score of $\geq 263 \mathrm{~dB} / \mathrm{m}$, the weight-gain groups had increased odds of NAFLD compared to the weight-stable (reference) group in the age- and sex-adjusted model (odds ratio [OR] $1.61,95 \%$ CI 1.07-2.43 for quartile 3; OR 3.58, 95\%CI 2.195.86 for quartile 4). After adjustment for age, sex, race/ ethnicity, education level, marital status, smoking status, hypertension, diabetes, total cholesterol, high-density lipoprotein cholesterol and physical activity, individuals who gained over $9.1 \mathrm{~kg}$ (20 pounds over 10 years) and composed quartile 4 had significantly greater odds of NAFLD (OR $2.63,95 \%$ CI 1.72-4.02). For every $\mathrm{kg}$ gained, the odds of NAFLD increased by 3\% (OR 1.03, 95\%CI 1.01-1.04), after adjustment for multiple confounders. The addition of waist circumference to the model reduced the OR for NAFLD, but it remained significant (OR 1.87, 95\%CI 1.19-2.95). This result suggests that the association between weight changes and NAFLD might be partly mediated by abdominal obesity. When sensitivity analyses were performed using a CAP score of $\geq 285 \mathrm{~dB} / \mathrm{m}$, similar and statistically significant associations between weight gain and NAFLD were observed in the univariate and multivariate models.

In terms of significant fibrosis (Table 3 ), the weight-gain groups had greater odds of significant fibrosis compared to the weight-stable group in the age- and sex-adjusted model (OR 2.42, 95\%CI 1.27-4.62 for quartile 3; OR 4.90, 95\%CI 2.52 9.56 for quartile 4). After adjustment for demographic and metabolic risk factors, the weight-gain groups had significantly greater odds of significant fibrosis compared to the weightstable group (OR 2.15, 95\%CI 1.19-3.87 for quartile 3; OR 3.73, 95\%CI 1.86-7.46 for quartile 4). For every $1 \mathrm{~kg}$ of weight gain, the odds of significant fibrosis increased by $3 \%$ (OR 1.03, 95\%CI 1.01-1.06). When waist circumference was included in the model, the association between weight changes and significant fibrosis was attenuated, but remained statistically significant. 
Table 1 Characteristics of the study population according to weight change over 10 years. $(n=2849)$

\begin{tabular}{|c|c|c|c|c|c|}
\hline \multirow[t]{2}{*}{ Characteristics } & \multicolumn{5}{|c|}{ Quartiles of weight change } \\
\hline & Quartile 1 & Quartile 2 & Quartile 3 & Quartile 4 & P-value \\
\hline Age (years) & $60.1 \pm 0.7$ & $58.8 \pm 0.7$ & $55.6 \pm 0.7$ & $51.8 \pm 0.6$ & $<0.001$ \\
\hline Sex (\% men) & $\begin{array}{c}52.4 \\
(44.4-60.1)\end{array}$ & $\begin{array}{c}49.9 \\
(43.0-56.7)\end{array}$ & $\begin{array}{c}44.5 \\
(37.2-52.1)\end{array}$ & $\begin{array}{c}38.9 \\
(33.5-44.7)\end{array}$ & 0.061 \\
\hline Body mass index $\left(\mathrm{kg} / \mathrm{m}^{2}\right)$ & $27.8 \pm 0.4$ & $27.3 \pm 0.3$ & $29.1 \pm 0.5$ & $34.1 \pm 0.3$ & $<0.001$ \\
\hline Waist circumference $(\mathrm{cm})$ & $98.5 \pm 0.9$ & $96.7 \pm 0.9$ & $100.6 \pm 1.5$ & $110.6 \pm 0.8$ & $<0.001$ \\
\hline Hypertension (\%) & $\begin{array}{c}51.6 \\
(45.1-58.0)\end{array}$ & $\begin{array}{c}37.7 \\
(32.2-43.5)\end{array}$ & $\begin{array}{c}42.1 \\
(36.1-48.4)\end{array}$ & $\begin{array}{c}44.2 \\
(38.8-49.8)\end{array}$ & 0.005 \\
\hline Diabetes (\%) & $\begin{array}{c}26.4 \\
(20.5-33.2)\end{array}$ & $\begin{array}{c}15.0 \\
(11.0-20.2)\end{array}$ & $\begin{array}{c}14.7 \\
(12.0-17.7)\end{array}$ & $\begin{array}{c}19.4 \\
(15.7-23.6)\end{array}$ & 0.002 \\
\hline $\begin{array}{l}\text { Ethnicity (\%) } \\
\text { Non-Hispanic White }\end{array}$ & $\begin{array}{c}69.3 \\
(60.6-76.8)\end{array}$ & $\begin{array}{c}68.8 \\
(61.8-74.9)\end{array}$ & $\begin{array}{c}64.9 \\
(59.4-70.1)\end{array}$ & $\begin{array}{c}61.3 \\
(53.2-68.8)\end{array}$ & 0.001 \\
\hline Non-Hispanic Black & $\begin{array}{c}9.9 \\
(6.6-14.6)\end{array}$ & $\begin{array}{c}5.5 \\
(3.5-8.5)\end{array}$ & $\begin{array}{c}9.7 \\
(6.8-13.6)\end{array}$ & $\begin{array}{c}16.1 \\
(11.5-22.1)\end{array}$ & \\
\hline Hispanic & $\begin{array}{c}11.3 \\
(8.1-15.5)\end{array}$ & $\begin{array}{c}12.3 \\
(9.2-16.3)\end{array}$ & $\begin{array}{c}15.4 \\
(11.4-20.4)\end{array}$ & $\begin{array}{c}14.1 \\
(10.6-18.9)\end{array}$ & \\
\hline Non-Hispanic Asian & $\begin{array}{c}4.1 \\
(2.8-5.9)\end{array}$ & $\begin{array}{c}9.1 \\
(5.9-13.7)\end{array}$ & $\begin{array}{c}7.2 \\
(4.7-10.7)\end{array}$ & $\begin{array}{c}3.5 \\
(2.4-5.2)\end{array}$ & \\
\hline Other & $\begin{array}{c}5.5 \\
(2.9-9.9)\end{array}$ & $\begin{array}{c}4.4 \\
(2.0-9.2)\end{array}$ & $\begin{array}{c}2.9 \\
(1.4-5.9)\end{array}$ & $\begin{array}{c}5.0 \\
(2.7-9.0)\end{array}$ & \\
\hline Smoking (\%) & & & & & 0.027 \\
\hline Never & $\begin{array}{c}52.0 \\
(47.0-57.0)\end{array}$ & $\begin{array}{c}62.5 \\
(56.6-68.1)\end{array}$ & $\begin{array}{c}62.0 \\
(55.7-67.9)\end{array}$ & $\begin{array}{c}54.4 \\
(49.0-60.0)\end{array}$ & \\
\hline Current smoker & $\begin{array}{c}29.4 \\
(25.9-33.1)\end{array}$ & $\begin{array}{c}26.0 \\
(20.6-31.3)\end{array}$ & $\begin{array}{c}27.7 \\
(23.6-32.2)\end{array}$ & $\begin{array}{c}31.9 \\
(26.4-38.0)\end{array}$ & \\
\hline Ex-smoker & $\begin{array}{c}18.6 \\
(15.0-22.8)\end{array}$ & $\begin{array}{c}11.9 \\
(7.6-18.2)\end{array}$ & $\begin{array}{c}10.3 \\
(7.0-14.9)\end{array}$ & $\begin{array}{c}13.7 \\
(10.8-17.2)\end{array}$ & \\
\hline High education (\%) & $\begin{array}{c}87.6 \\
(84.4-90.1)\end{array}$ & $\begin{array}{c}90.6 \\
(86.7-93.5)\end{array}$ & $\begin{array}{c}91.0 \\
(88.1-93.2)\end{array}$ & $\begin{array}{c}88.0 \\
(84.6-90.6)\end{array}$ & 0.170 \\
\hline Married (\%) & $\begin{array}{c}68.7 \\
(63.2-73.8)\end{array}$ & $\begin{array}{c}69.1 \\
(62.2-75.2)\end{array}$ & $\begin{array}{c}74.2 \\
(70.1-77.9)\end{array}$ & $\begin{array}{c}67.3 \\
(61.1-73.0)\end{array}$ & 0.304 \\
\hline Total cholesterol (mg/dL) & $187.2 \pm 3.0$ & $197.2 \pm 3.5$ & $198.2 \pm 3.3$ & $198.9 \pm 2.7$ & 0.003 \\
\hline High-density lipoprotein cholesterol (mg/dL) & $55.0 \pm 1.3$ & $56.6 \pm 1.3$ & $54.2 \pm 1.0$ & $50.7 \pm 0.8$ & 0.002 \\
\hline Triglyceride (mg/dL) & $119.6 \pm 5.1$ & $112.2 \pm 6.9$ & $111.6 \pm 3.2$ & $135.2 \pm 9.8$ & 0.149 \\
\hline Fasting glucose (mg/dL) & $119.6 \pm 4.9$ & $110.7 \pm 1.4$ & $110.4 \pm 1.6$ & $114.1 \pm 2.1$ & 0.265 \\
\hline Hemoglobin A1c (\%) & $5.92 \pm 0.06$ & $5.71 \pm 0.05$ & $5.75 \pm 0.04$ & $5.88 \pm 0.04$ & 0.952 \\
\hline Fasting insulin (pmol/L) & $70.3 \pm 7.5$ & $65.5 \pm 5.8$ & $79.4 \pm 8.4$ & $98.2 \pm 7.3$ & 0.021 \\
\hline Alanine aminotransferase (IU/L) & $19.0 \pm 0.6$ & $20.6 \pm 0.5$ & $21.4 \pm 0.7$ & $24.8 \pm 0.8$ & $<0.001$ \\
\hline Aspartate aminotransferase (IU/L) & $20.9 \pm 0.5$ & $21.1 \pm 0.3$ & $21.3 \pm 0.5$ & $21.2 \pm 0.3$ & 0.565 \\
\hline Gamma glutamyl transferase (IU/L) & $25.7 \pm 1.5$ & $25.4 \pm 1.1$ & $28.4 \pm 1.1$ & $32.0 \pm 1.0$ & 0.006 \\
\hline NAFLD (CAP $\geq 263 \mathrm{~dB} / \mathrm{m}, \%)$ & $\begin{array}{c}48.4 \\
(40.2-56.7)\end{array}$ & $\begin{array}{c}44.4 \\
(38.4-50.6)\end{array}$ & $\begin{array}{c}53.3 \\
(46.5-60.1)\end{array}$ & $\begin{array}{c}68.8 \\
(61.5-75.3)\end{array}$ & $<0.001$ \\
\hline NAFLD (CAP $\geq 285$ dB/m, \%) & $\begin{array}{c}35.0 \\
(28.8-41.8)\end{array}$ & $\begin{array}{c}29.1 \\
(23.9-35.6)\end{array}$ & $\begin{array}{c}35.8 \\
(28.6-43.8)\end{array}$ & $\begin{array}{c}56.5 \\
(50.0-62.8)\end{array}$ & $<0.001$ \\
\hline Significant fibrosis $(\geq \mathrm{F} 2, \%)$ & $\begin{array}{c}8.9 \\
(5.9-13.0)\end{array}$ & $\begin{array}{c}3.9 \\
(2.3-6.4)\end{array}$ & $\begin{array}{c}8.1 \\
(6.1-10.8)\end{array}$ & $\begin{array}{c}13.6 \\
(10.7-17.2)\end{array}$ & $<0.001$ \\
\hline
\end{tabular}

Data are shown as the weighted mean \pm standard error or weighted frequency ( $95 \%$ confidence interval), as appropriate

Triglycerides, fasting glucose, fasting insulin were calculated from fasting subjects only

Range of quartile $1,<-7(-3.18 \mathrm{~kg})$ pounds; quartile $2,-7$ pounds $(-3.18 \mathrm{~kg})$ to $6(2.72 \mathrm{~kg})$ pounds; quartile 3,6 pounds $(2.72 \mathrm{~kg})$ to 20 pounds; and quartile 4 , $\geq 20$ pounds $(9.07 \mathrm{~kg})$

CAP, controlled attenuation parameter; NAFLD, nonalcoholic fatty liver disease 
Table 2 Multivariate odds ratio for NAFLD based on weight changes over 10 years

\begin{tabular}{|c|c|c|c|c|c|c|}
\hline \multirow[t]{2}{*}{ Quartile } & \multicolumn{2}{|c|}{ Age, sex-adjusted model } & \multicolumn{2}{|c|}{ Multivariate model 1} & \multicolumn{2}{|c|}{ Multivariate model 2} \\
\hline & OR $(95 \% \mathrm{CI})$ & P-value & OR $(95 \% \mathrm{CI})$ & $\mathrm{P}$-value & OR $(95 \% \mathrm{CI})$ & P-value \\
\hline \multicolumn{7}{|c|}{ NAFLD $(C A P \geq 263 \mathrm{~dB} / \mathrm{m})$} \\
\hline $1^{\text {st }}$ quartile & $1.13(0.73-1.73)$ & 0.562 & $0.84(0.52-2.03)$ & 0.216 & $0.94(0.58-1.51)$ & 0.782 \\
\hline $2^{\text {nd }}$ quartile & 1 & & 1 & & 1 & \\
\hline $3^{\text {rd }}$ quartile & $1.61(1.07-2.43)$ & 0.026 & $1.31(0.84-2.03)$ & 0.216 & $1.24(0.78-1.96)$ & 0.344 \\
\hline $4^{\text {th }}$ quartile & $3.58(2.19-5.86)$ & $<0.001$ & $2.63(1.72-4.02)$ & $<0.001$ & $1.87(1.19-2.95)$ & 0.010 \\
\hline Per $1 \mathrm{~kg}$ increase & $1.03(1.01-1.05)$ & 0.001 & $1.03(1.01-1.04)$ & 0.005 & $1.01(1.00-1.03)$ & 0.041 \\
\hline \multicolumn{7}{|c|}{ NAFLD $(C A P \geq 285 \mathrm{~dB} / \mathrm{m})$} \\
\hline $1^{\text {st }}$ quartile & $1.26(0.81-1.97)$ & 0.280 & $1.00(0.64-1.57)$ & 0.993 & $1.14(0.77-1.68)$ & 0.492 \\
\hline $2^{\text {nd }}$ quartile & 1 & & 1 & & 1 & \\
\hline $3^{\text {rd }}$ quartile & $1.51(0.96-2.39)$ & 0.074 & $1.24(0.82-1.85)$ & 0.282 & $1.14(0.77-1.68)$ & 0.492 \\
\hline $4^{\text {th }}$ quartile & $4.05(2.54-6.44)$ & $<0.001$ & $3.02(2.03-4.48)$ & $<0.001$ & $2.23(1.48-3.35)$ & 0.001 \\
\hline Per $1 \mathrm{~kg}$ increase & $1.03(1.02-1.04)$ & $<0.001$ & $1.02(1.01-1.04)$ & $<0.001$ & $1.01(1.00-1.02)$ & 0.009 \\
\hline
\end{tabular}

Multivariate model 1 was adjusted for age, sex, race/ethnicity, education level, marital status, smoking status, hypertension, diabetes, total cholesterol, highdensity lipoprotein cholesterol, and leisure-time physical activity, using appropriate sampling weights

Multivariate model 2 was adjusted for waist circumference in addition to model 1, using appropriate sampling weights

Range of quartile 1, <-7 (-3.18 kg) pounds; quartile 2, -7 pounds $(-3.18 \mathrm{~kg})$ to $6(2.72 \mathrm{~kg})$ pounds; quartile 3,6 pounds $(2.72 \mathrm{~kg})$ to 20 pounds; and quartile 4 , $\geq 20$ pounds $(9.07 \mathrm{~kg})$

CAP, controlled attenuation parameter; NAFLD, nonalcoholic fatty liver disease; OR, odds ratio; CI, confidence interval

Table 3 Multivariate odds ratio for significant fibrosis based on weight change over 10 years

\begin{tabular}{|c|c|c|c|c|c|c|}
\hline \multirow[t]{2}{*}{ Quartile } & \multicolumn{2}{|c|}{ Age, sex-adjusted model } & \multicolumn{2}{|c|}{ Multivariate model 1} & \multicolumn{2}{|c|}{ Multivariate model 2} \\
\hline & OR $(95 \% \mathrm{CI})$ & P-value & OR $(95 \% \mathrm{CI})$ & P-value & OR $(95 \% \mathrm{CI})$ & $\mathrm{P}$-value \\
\hline \multicolumn{7}{|l|}{ Total population } \\
\hline $1^{\text {st }}$ quartile & $2.32(1.15-4.71)$ & 0.023 & $1.57(0.81-3.04)$ & 0.166 & $1.62(0.83-3.16)$ & 0.144 \\
\hline $2^{\text {nd }}$ quartile & 1 & & 1 & & 1 & \\
\hline $3^{\text {rd }}$ quartile & $2.42(1.27-4.62)$ & 0.011 & $2.15(1.19-3.87)$ & 0.014 & $1.99(1.05-3.79)$ & 0.037 \\
\hline $4^{\text {th }}$ quartile & $4.90(2.52-9.56)$ & $<0.001$ & $3.73(1.86-7.46)$ & 0.001 & $3.12(1.46-6.65)$ & 0.006 \\
\hline Per $1 \mathrm{~kg}$ increase & $1.04(1.02-1.06)$ & 0.001 & $1.03(1.01-1.06)$ & 0.005 & $1.03(1.00-1.05)$ & 0.023 \\
\hline \multicolumn{7}{|l|}{ Among subjects with } \\
\hline \multicolumn{7}{|c|}{ NAFLD $(C A P \geq 263 \mathrm{~dB} / \mathrm{m})$} \\
\hline $1^{\text {st }}$ quartile & $1.67(0.77-3.63)$ & 0.180 & $1.35(0.61-3.00)$ & 0.431 & $1.31(0.58-2.98)$ & 0.492 \\
\hline $2^{\text {nd }}$ quartile & 1 & & 1 & & 1 & \\
\hline $3^{\text {rd }}$ quartile & $1.95(0.89-4.24)$ & 0.088 & $1.82(0.93-3.54)$ & 0.075 & $1.72(0.82-3.61)$ & 0.143 \\
\hline $4^{\text {th }}$ quartile & $3.14(1.48-6.67)$ & 0.005 & $2.64(1.30-5.36)$ & 0.010 & $2.39(1.10-5.19)$ & 0.030 \\
\hline Per $1 \mathrm{~kg}$ increase & $1.03(1.01-1.06)$ & 0.017 & $1.03(1.00-1.06)$ & 0.042 & $1.03(1.00-1.06)$ & 0.058 \\
\hline \multicolumn{7}{|l|}{ Among subjects with } \\
\hline \multicolumn{7}{|c|}{ NAFLD $($ CAP $\geq 285 \mathrm{~dB} / \mathrm{m})$} \\
\hline $1^{\text {st }}$ quartile & $1.48(0.73-2.97)$ & 0.253 & $1.25(0.55-2.85)$ & 0.565 & $1.22(0.52-2.85)$ & 0.624 \\
\hline $2^{\text {nd }}$ quartile & 1 & & 1 & & 1 & \\
\hline $3^{\text {rd }}$ quartile & $1.76(0.81-3.83)$ & 0.140 & $1.61(0.87-2.99)$ & 0.120 & $1.55(0.78-3.11)$ & 0.195 \\
\hline $4^{\text {th }}$ quartile & $2.48(1.23-4.97)$ & 0.014 & $2.36(1.17-4.74)$ & 0.019 & $2.21(1.02-4.80)$ & 0.045 \\
\hline Per $1 \mathrm{~kg}$ increase & $1.04(1.01-1.06)$ & 0.004 & $1.03(1.01-1.06)$ & 0.011 & $1.03(1.01-1.06)$ & 0.017 \\
\hline
\end{tabular}

Multivariate model 1 was adjusted for age, sex, race/ethnicity, education level, marital status, smoking status, hypertension, diabetes, total cholesterol, highdensity lipoprotein-cholesterol, and leisure-time physical activity, using appropriate sampling weights

Multivariate model 2 was adjusted for waist circumference in addition to model 1, using appropriate sampling weights

Range of quartile $1,<-7(-3.18 \mathrm{~kg})$ pounds; quartile 2, -7 pounds $(-3.18 \mathrm{~kg})$ to $6(2.72 \mathrm{~kg})$ pounds; quartile 3,6 pounds $(2.72 \mathrm{~kg})$ to 20 pounds; and quartile 4 , $\geq 20$ pounds $(9.07 \mathrm{~kg})$

CAP, controlled attenuation parameter; NAFLD, nonalcoholic fatty liver disease; OR, odds ratio; CI, confidence interval

Sensitivity analyses were conducted among individuals with NAFLD using the different definitions of NAFLD based on different CAP score cutoff. Overall, the results were identical to those obtained using the cohort. 


\section{Weight changes over 1 year and NAFLD/significant fibrosis}

As shown in Supplementary Table 1, individuals in the weight-gain groups (quartiles 3 and 4 ) tended to be younger, more obese, female, Hispanic, non-Hispanic Black, current or ex-smokers, and more likely to have higher levels of liver enzymes compared to individuals in the weight-stable group. Consistent with previous results using weight changes over 10 years, the prevalence of NAFLD in the weight-gain group was higher than that in the weight-stable group, regardless of differences in the definition of NAFLD. Results of logistic regression analyses are presented in Table 4. Individuals who gained more than $1.81 \mathrm{~kg}$ (4 pounds; quartile 4) over a year had significantly greater odds of NAFLD (OR 3.55, 95\%CI 2.31$5.47)$ in the age- and sex-adjusted model. When we considered demographic and metabolic risk factors (model 1) as well as waist circumference (model 2), the association between weight changes and NAFLD remained statistically significant (OR 3.91, 95\%CI 2.32-6.58 for model 1; OR 3.15, 95\%CI 1.765.67 for model 2). In addition, the odds of NAFLD increased significantly with every 1 -kg increase over a year (OR 1.04, 95\%CI 1.01-1.06). Sensitivity analyses were performed using a CAP score of $\geq 285 \mathrm{~dB} / \mathrm{m}$. Overall, the results were similar to those obtained using a CAP score of $\geq 263 \mathrm{~dB} / \mathrm{m}$. Regarding significant fibrosis in the total population (Table 5), individuals who gained more than $1.81 \mathrm{~kg}$ (4 pounds; quartile 4) over a year had greater odds of significant fibrosis in the age- and sex-adjusted model and multivariate model 1 . However, this association became non-significant after waist circumference was taken into consideration. In addition, the odds of significant fibrosis were not linearly associated with weight gain (per 1-kg increase). Among individuals with NAFLD, weight gain of more than $1.81 \mathrm{~kg}$ (4 pounds; quartile 4) over a year was not associated with significant fibrosis in the age- and sex-adjusted model or in the multivariate models, regardless of the definition of NAFLD.

\section{Discussion}

This study demonstrates the impact of weight gain over the short (1 year) and long (10 year) term on the risk of NAFLD and significant fibrosis, as measured by transient elastography. The current study found that adults who gained more than $9.07 \mathrm{~kg}$ (20 pounds) over 10 years had over 2 -fold greater odds of NAFLD and an approximately 3 -fold greater odds of significant fibrosis than those with stable weights. We found that weight gain over 10 years was associated with a greater risk of NAFLD and NAFLD-associated significant fibrosis, independently of demographic and metabolic risk factors. In addition, individuals who gained at least $1.81 \mathrm{~kg}$ (4 pounds) over a year had over 3-fold greater odds of NAFLD compared with those with stable weights.

A previous meta-analysis demonstrated a positive association between obesity and risk of NAFLD [21]. Several studies have demonstrated an association between weight gain, NAFLD and liver fibrosis in the Asian population $[8,22,23]$. Another study that evaluated 88 biopsyproven patients with NAFLD over a 1-year follow up showed fibrosis progression in those with significant weight gain, defined as more than $5 \mathrm{~kg}$ [24]. The current study used a lower cutoff ( $1.81 \mathrm{~kg}, 4$ pounds) over one year and did not demonstrate a significant association between weight gain and significant fibrosis. This may be explained by the fact

Table 4 Multivariate odds ratio for NAFLD based on weight change over 1 year

\begin{tabular}{|c|c|c|c|c|c|c|}
\hline \multirow[t]{2}{*}{ Quartile } & \multicolumn{2}{|c|}{ Age, sex-adjusted model } & \multicolumn{2}{|c|}{ Multivariate model 1} & \multicolumn{2}{|c|}{ Multivariate model 2} \\
\hline & OR $(95 \% \mathrm{CI})$ & P-value & OR $(95 \% \mathrm{CI})$ & P-value & OR $(95 \% \mathrm{CI})$ & P-value \\
\hline \multicolumn{7}{|c|}{ NAFLD $(\mathrm{CAP} \geq 263 \mathrm{~dB} / \mathrm{m})$} \\
\hline $1^{\text {st }}$ quartile & $2.11(1.40-3.19)$ & 0.002 & $1.83(1.25-2.68)$ & 0.004 & $1.45(0.95-2.21)$ & 0.081 \\
\hline $2^{\text {nd }}$ quartile & 1 & & 1 & & 1 & \\
\hline $3^{\text {rd }}$ quartile & $1.71(1.00-2.91)$ & 0.049 & $1.78(1.02-3.09)$ & 0.041 & $1.63(0.84-3.18)$ & 0.139 \\
\hline $4^{\text {th }}$ quartile & $3.55(2.31-5.47)$ & $<0.001$ & $3.91(2.32-6.58)$ & $<0.001$ & $3.15(1.76-5.67)$ & 0.001 \\
\hline Per $1 \mathrm{~kg}$ increase & $1.02(1.00-1.04)$ & 0.029 & $1.04(1.01-1.06)$ & 0.006 & $1.04(1.01-1.06)$ & 0.005 \\
\hline \multicolumn{7}{|c|}{ NAFLD $(\mathrm{CAP} \geq 285 \mathrm{~dB} / \mathrm{m})$} \\
\hline $1^{\text {st }}$ quartile & $1.93(1.10-3.39)$ & 0.014 & $1.43(0.88-2.31)$ & 0.136 & $1.12(0.69-1.81)$ & 0.621 \\
\hline $2^{\text {nd }}$ quartile & 1 & & 1 & & 1 & \\
\hline $3^{\text {rd }}$ quartile & $1.57(0.95-2.62)$ & 0.076 & $1.40(0.82-2.39)$ & 0.198 & $1.26(0.67-2.35)$ & 0.445 \\
\hline $4^{\text {th }}$ quartile & $2.77(1.69-4.53)$ & 0.001 & $2.46(1.50-4.03)$ & 0.002 & $1.91(1.12-3.26)$ & 0.021 \\
\hline
\end{tabular}

Multivariate model 1 was adjusted for age, sex, Race/ethnicity, education level, marital status, smoking status, hypertension, diabetes, total cholesterol, highdensity lipoprotein-cholesterol, and leisure-time physical activity, using appropriate sampling weights

Multivariate model 2 was adjusted for waist circumference in addition to model 1, using appropriate sampling weights

Range of quartile $1,<-5$ pounds $(-2.27 \mathrm{~kg})$; quartile $2,-5$ pounds $(-2.27 \mathrm{~kg})$ to 0 pounds; quartile 3,0 pounds to 4 pounds (1.81 kg); and quartile $4, \geq 4$ pounds $(1.81 \mathrm{~kg})$

CAP, controlled attenuation parameter; NAFLD, nonalcoholic fatty liver disease; OR, odds ratio; CI, confidence interval 
Table 5 Multivariate odds ratio for significant fibrosis based on weight change over 1 year

\begin{tabular}{|c|c|c|c|c|c|c|}
\hline \multirow[t]{2}{*}{ Quartile } & \multicolumn{2}{|c|}{ Age, sex-adjusted model } & \multicolumn{2}{|c|}{ Multivariate model 1} & \multicolumn{2}{|c|}{ Multivariate model 2} \\
\hline & OR $(95 \% \mathrm{CI})$ & P-value & OR $(95 \% \mathrm{CI})$ & P-value & OR $(95 \% \mathrm{CI})$ & P-value \\
\hline \multicolumn{7}{|l|}{ Total population } \\
\hline $1^{\text {st }}$ quartile & $1.95(0.91-4.18)$ & 0.084 & $1.61(0.66-3.89)$ & 0.270 & $1.41(0.55-3.65)$ & 0.450 \\
\hline $2^{\text {nd }}$ quartile & 1 & & 1 & & 1 & \\
\hline $3^{\text {rd }}$ quartile & $1.31(0.62-2.76)$ & 0.448 & $1.29(0.52-3.20)$ & 0.553 & $1.21(0.45-3.27)$ & 0.687 \\
\hline $4^{\text {th }}$ quartile & $2.53(1.30-4.93)$ & 0.009 & $2.17(1.11-4.23)$ & 0.026 & $1.82(0.86-3.85)$ & 0.108 \\
\hline Per $1 \mathrm{~kg}$ increase & $1.01(0.98-1.04)$ & 0.630 & $1.01(0.98-1.03)$ & 0.578 & $1.01(0.98-1.03)$ & 0.573 \\
\hline \multicolumn{7}{|c|}{ Among subjects with } \\
\hline \multicolumn{7}{|c|}{ NAFLD $(C A P \geq 263 \mathrm{~dB} / \mathrm{m})$} \\
\hline $1^{\text {st }}$ quartile & $1.34(0.48-3.73)$ & 0.553 & $1.18(0.38-3.63)$ & 0.763 & $1.13(0.35-3.66)$ & 0.824 \\
\hline $2^{\text {nd }}$ quartile & 1 & & 1 & & 1 & \\
\hline $3^{\text {rd }}$ quartile & $1.03(0.40-2.64)$ & 0.944 & $1.06(0.34-3.28)$ & 0.916 & $1.08(0.33-3.47)$ & 0.895 \\
\hline $4^{\text {th }}$ quartile & $1.20(0.55-2.59)$ & 0.629 & $1.25(0.50-3.12)$ & 0.613 & $1.18(0.44-3.14)$ & 0.728 \\
\hline Per $1 \mathrm{~kg}$ increase & $1.01(0.97-1.05)$ & 0.522 & $1.00(0.98-1.03)$ & 0.845 & $1.00(0.98-1.03)$ & 0.851 \\
\hline \multicolumn{7}{|c|}{$\begin{array}{l}\text { Among subjects with } \\
\text { NAFLD }(C A P \geq 285 \mathrm{~dB} / \mathrm{m})\end{array}$} \\
\hline $1^{\text {st }}$ quartile & $1.16(0.37-3.58)$ & 0.788 & $1.11(0.33-3.78)$ & 0.851 & $1.11(0.32-3.84)$ & 0.858 \\
\hline $2^{\text {nd }}$ quartile & 1 & & 1 & & 1 & \\
\hline $3^{\text {rd }}$ quartile & $1.00(0.37-2.69)$ & 0.997 & $1.07(0.33-3.43)$ & 0.906 & $1.12(0.35-3.61)$ & 0.841 \\
\hline $4^{\text {th }}$ quartile & $1.19(0.52-2.75)$ & 0.659 & $1.35(0.49-3.71)$ & 0.535 & $1.34(0.47-3.80)$ & 0.555 \\
\hline Per $1 \mathrm{~kg}$ increase & $1.02(0.98-1.05)$ & 0.277 & $1.01(0.98-1.04)$ & 0.419 & $1.01(0.99-1.04)$ & 0.411 \\
\hline
\end{tabular}

Multivariate model 1 was adjusted for age, sex, Race/ethnicity, education level, marital status, smoking status, hypertension, diabetes, total cholesterol, highdensity lipoprotein-cholesterol, and leisure-time physical activity, using appropriate sampling weights

Multivariate model 2 was adjusted for waist circumference in addition to model 1, using appropriate sampling weights

Range of quartile $1,<-5$ pounds $(-2.27 \mathrm{~kg})$; quartile $2,-5$ pounds $(-2.27 \mathrm{~kg})$ to 0 pounds; quartile 3,0 pounds to 4 pounds (1.81 kg); and quartile $4, \geq 4$ pounds $(1.81 \mathrm{~kg})$

CAP, controlled attenuation parameter; NAFLD, nonalcoholic fatty liver disease; OR, odds ratio; CI, confidence interval

that significant liver fibrosis takes time to progress or regress. However, our study found that weight gain over 10 years may increase the risk of NAFLD and significant fibrosis in a representative sample of the US general population, which is consistent with prior studies [8,21-24]. The fibrosis stage, not NASH, has been shown to predict mortality and the time for developing advanced liver disease in NAFLD [9]. As there are no approved pharmacologic treatments for NAFLD or fibrosis, avoiding weight gain may be another preventive option for NAFLD and significant fibrosis [25]. Thus, interventions aimed to prevent significant weight gain over a long-term period may prevent advanced liver disease in the general population and those with NAFLD.

Previous studies support the association between weight gain and the development of liver fibrosis and NAFLD [12,26-28]. Studies have demonstrated that shortterm weight gain is an independent risk factor for NAFLD among nonobese Asian individuals [12,27]. However, no prior studies have investigated the long-term effect of weight gain in non-Asian populations. A recent prospective cohort study from Korea with a median follow up of 6 years reported that obesity and weight gain were associated with progression of liver fibrosis, assessed using the aspartate aminotransferase to platelet ratio index (APRI) [8]. However, that study had some limitations. The investigators used ultrasound for the diagnosis of NAFLD and APRI for the assessment of liver fibrosis. It has been shown that transient elastography is superior to ultrasound for detecting mild hepatic steatosis ( $>5 \%$ of hepatocytes) and superior to APRI for detecting significant fibrosis $[29,30]$. Thus, we used transient elastography in our study to diagnose NAFLD and significant fibrosis. In addition, the current study has longterm data on weight gain (over 10 years), which is a longer follow up than that in the recent prospective Korean study (6 years). Most of the studies that reported an association between weight gain, NAFLD and significant fibrosis were conducted in Asia $(8,12,27)$. The current study used population-based data from the US, representing a more diverse race/ethnicity cohort.

The underlying mechanism behind the association between weight gain, NAFLD and liver fibrosis progression is not clearly understood. However, it is thought to be due to insulin resistance, inflammation, oxidative stress, and lipotoxicity that links to the progression of liver fibrosis [17,31-33]. However, one study showed a persistent, significant association between weight gain and noninvasive fibrosis assessment in NAFLD, after considering insulin resistance and high-sensitivity C-reactive protein [8]. This suggests multiple complex pathogenic mechanisms behind the association between weight gain and fibrosis in NAFLD. 
Some adipokines, such as leptin and adiponectin, may play a role in the pathogenesis of liver fibrosis progression in NAFLD [17]. Leptin is a pro-fibrogenic cytokine, while adiponectin has anti-fibrogenic and anti-inflammatory properties [34]. Thus, dysregulation of these adipokines may explain the risk of significant fibrosis in individuals with obesity and NAFLD [31,34,35].

The current study has several strengths. We used the CAP score to define NAFLD. The CAP score is a parameter with high accuracy for mild hepatic steatosis ( $>5 \%$ of hepatocytes) and has a higher sensitivity in identifying NAFLD than ultrasonography or noninvasive serum panels for NAFLD [36]. In addition, we defined significant fibrosis using transient elastography, not noninvasive serum panels [29,30]. To date, there have been no prior studies focusing on the US population that have investigated the association between weight changes over 10 years, NAFLD and significant fibrosis, measured by transient elastography. Therefore, the findings of this study may be generalizable to the Western world, which shares similar demographic, lifestyle, and behavioral health patterns with those of the US.

The current study has also several limitations. First, we used a weight history questionnaire, which is at risk of recall bias. Second, this study cannot offer definitive conclusions regarding causality. Third, we were unable to obtain transient elastography data at baseline prior to 1 and 10 years. Therefore, we were not able to provide NAFLD and significant fibrosis status at 1 and 10 years before, although we excluded participants with a medical history of fatty liver and liver fibrosis/cirrhosis. This may explain why weight loss was not inversely associated with NAFLD and significant fibrosis. The weight-loss group had a higher prevalence of diabetes, hypertension, NAFLD and significant fibrosis, and was older than the weight-stable group. In addition, because of limitations in the NHANES dataset, we were unable to obtain information regarding extrahepatic cholestasis or systemic diseases affecting the liver, which may be uncommon in the general population. Fourth, there is no universal cutoff to determine NAFLD using the CAP score or to determine significant fibrosis in NAFLD using liver stiffness by transient elastography. However, we used validated cutoff points for the CAP score and liver stiffness, based on previously published studies $[10,19,20]$. Fifth, our study design meant we were unable to retrieve medications that may affect the prevalence or the progression of NAFLD. Future studies will be needed to determine the impact of medication on the association between weight gain and NAFLD. Moreover, because of the complex survey design applied by the NHANES, we were unable to perform a post hoc analysis after adjustment for appropriate sample weights.

In conclusion, weight gain over 10 years is associated with greater odds of NAFLD and significant fibrosis in US adults, independently of known demographic and metabolic risk factors. In addition, weight gain over a year is closely associated with an increase in the odds of NAFLD.

\section{Summary Box}

\section{What is already known:}

- Studies have reported an association between weight gain, nonalcoholic fatty liver disease (NAFLD), and liver fibrosis in the Asian population

- No studies have evaluated the impact of long-term weight gain on the risk of NAFLD and fibrosis in the Western population

- There is an urgent need to understand modifiable risk factors for NAFLD to reduce disease burden and associated healthcare costs

\section{What the new findings are:}

- This cross-sectional study showed that weight gain over 10 years was associated with a greater risk of NAFLD and significant fibrosis in United States adults

- Weight gain over a year was closely associated with an increase in the odds of NAFLD

\section{References}

1. Younossi ZM, Koenig AB, Abdelatif D, Fazel Y, Henry L, Wymer M. Global epidemiology of nonalcoholic fatty liver disease - metaanalytic assessment of prevalence, incidence, and outcomes. Hepatology 2016;64:73-84.

2. Unalp-Arida A, Ruhl CE. Noninvasive fatty liver markers predict liver disease mortality in the U.S. population. Hepatology 2016;63:1170-1183.

3. Kim D, Kim WR, Kim HJ, Therneau TM. Association between noninvasive fibrosis markers and mortality among adults with nonalcoholic fatty liver disease in the United States. Hepatology 2013;57:1357-1365.

4. Kim D, Cholankeril G, Li AA, et al. Trends in hospitalizations for chronic liver disease-related liver failure in the United States, 20052014. Liver Int 2019;39:1661-1671.

5. Murag S, Ahmed A, Kim D. Recent epidemiology of nonalcoholic fatty liver disease. Gut Liver 2021;15:206-216.

6. Paik JM, Golabi P, Younossi Y, Mishra A, Younossi ZM. Changes in the global burden of chronic liver diseases from 2012 to 2017: the growing impact of NAFLD. Hepatology 2020;72:1605-1616.

7. Polyzos SA, Kountouras J, Mantzoros CS. Obesity and nonalcoholic fatty liver disease: from pathophysiology to therapeutics. Metabolism 2019;92:82-97.

8. Kim Y, Chang Y, Cho YK, Ahn J, Shin H, Ryu S. Obesity and weight gain are associated with progression of fibrosis in patients with nonalcoholic fatty liver disease. Clin Gastroenterol Hepatol 2019; 17:543-550.

9. Hagström H, Nasr P, Ekstedt M, et al. Fibrosis stage but not NASH predicts mortality and time to development of severe liver disease in biopsy-proven NAFLD. J Hepatol 2017;67:1265-1273.

10. Eddowes PJ, Sasso M, Allison M, et al. Accuracy of FibroScan controlled attenuation parameter and liver stiffness measurement in assessing steatosis and fibrosis in patients with nonalcoholic 
fatty liver disease. Gastroenterology 2019;156:1717-1730.

11. Koutoukidis DA, Astbury NM, Tudor KE, et al. Association of weight loss interventions with changes in biomarkers of nonalcoholic fatty liver disease: a systematic review and metaanalysis. JAMA Intern Med 2019;179:1262-1271.

12. Yamada G, Hagiwara Y, Kimura T, et al. Impact of body weight gain on the incidence of nonalcoholic fatty liver disease in nonobese Japanese individuals. Am J Gastroenterol 2021;116:733-740.

13. CDC National Health and Nutrition Examination Survey (NHANES). Liver ultrasound transient elastography procedures manual. 2018. Available from: https://wwwn.cdc.gov/nchs/ data/nhanes/2017-2018/manuals/2018_Liver_Ultrasound_ Elastography_Procedures_Manual.pdf [Accessed 15 January 2022].

14. Kim D, Cholankeril G, Loomba R, Ahmed A. Prevalence of fatty liver disease and fibrosis detected by fibroscan in adults in the United States, 2017-2018. Clin Gastroenterol Hepatol 2020;19:14991501.e2.

15. Kim D, Cholankeril G, Loomba R, Ahmed A. Prevalence of nonalcoholic fatty liver disease and hepatic fibrosis among US adults with prediabetes and diabetes, NHANES 2017-2018. J Gen Intern Med 2022;37:261-263.

16. CDC National Health and Nutrition Examination Survey. 20172018 Data documentation, codebook, and frequencies. Liver ultrasound transient elastography (LUX_J). 2020. Available from: https://wwwn.cdc.gov/Nchs/Nhanes/2017-2018/LUX_J.htm [Accessed 15 January 2022].

17. Tilg H. Adipocytokines in nonalcoholic fatty liver disease: key players regulating steatosis, inflammation and fibrosis. Curr Pharm Des 2010;16:1893-1895.

18. Siddiqui MS, Vuppalanchi R, Van Natta ML, et al; NASH Clinical Research Network. Vibration-controlled transient elastography to assess fibrosis and steatosis in patients with nonalcoholic fatty liver disease. Clin Gastroenterol Hepatol 2019;17:156-163.

19. Castera L, Friedrich-Rust M, Loomba R. Noninvasive assessment of liver disease in patients with nonalcoholic fatty liver disease. Gastroenterology 2019;156:1264-1281.

20. Abeysekera KWM, Fernandes GS, Hammerton G, et al. Prevalence of steatosis and fibrosis in young adults in the UK: a populationbased study. Lancet Gastroenterol Hepatol 2020;5:295-305.

21. Li L, Liu DW, Yan HY, Wang ZY, Zhao SH, Wang B. Obesity is an independent risk factor for non-alcoholic fatty liver disease: evidence from a meta-analysis of 21 cohort studies. Obes Rev 2016;17:510-519.

22. Du S, Wang C, Jiang W, et al. The impact of body weight gain on nonalcoholic fatty liver disease and metabolic syndrome during earlier and later adulthood. Diabetes Res Clin Pract 2016;116:183-191.

23. Zhang WJ, Chen LL, Zheng J, Lin L, Zhang JY, Hu X. Association of adult weight gain and nonalcoholic fatty liver in a cross-sectional study in Wan Song Community, China. Braz J Med Biol Res 2014;47:151-156.

24. Ekstedt M, Franzén LE, Mathiesen UL, et al. Long-term follow-up of patients with NAFLD and elevated liver enzymes. Hepatology 2006;44:865-873.

25. Romero-Gómez M, Zelber-Sagi S, Trenell M. Treatment of NAFLD with diet, physical activity and exercise. J Hepatol 2017;67:829-846.

26. Ratziu V, Giral P, Charlotte F, et al. Liver fibrosis in overweight patients. Gastroenterology 2000;118:1117-1123.

27. Liu CJ. Prevalence and risk factors for non-alcoholic fatty liver disease in Asian people who are not obese. J Gastroenterol Hepatol 2012;27:1555-1560.

28. Ting YW, Wong SW, Anuar Zaini A, Mohamed R, Jalaludin MY. Metabolic syndrome is associated with advanced liver fibrosis among pediatric patients with non-alcoholic fatty liver disease. Front Pediatr 2019;7:491.

29. Göbel T, Schadewaldt-Tümmers J, Greiner L, Poremba C, Häussinger D, Erhardt A. Transient elastography improves detection of liver cirrhosis compared to routine screening tests. World J Gastroenterol 2015;21:953-960.

30. Zhang GL, Zhao QY, Lin CS, Hu ZX, Zhang T, Gao ZL. Transient elastography and ultrasonography: optimal evaluation of liver fibrosis and cirrhosis in patients with chronic hepatitis B concurrent with nonalcoholic fatty liver disease. Biomed Res Int 2019;2019:3951574.

31. Fabbrini E, Sullivan S, Klein S. Obesity and nonalcoholic fatty liver disease: biochemical, metabolic, and clinical implications. Hepatology 2010;51:679-689.

32. Neuschwander-Tetri BA. Hepatic lipotoxicity and the pathogenesis of nonalcoholic steatohepatitis: the central role of nontriglyceride fatty acid metabolites. Hepatology 2010;52:774-788.

33. Angulo P, Machado MV, Diehl AM. Fibrosis in nonalcoholic fatty liver disease: mechanisms and clinical implications. Semin Liver Dis 2015;35:132-145.

34. Park PH, Sanz-Garcia C, Nagy LE. Adiponectin as an anti-fibrotic and anti-inflammatory adipokine in the liver. Curr Pathobiol Rep 2015;3:243-252.

35. Landecho MF, Tuero C, Valenti V, Bilbao I, de Higuera, Fruhbeck G. Relevance of leptin and other adipokines in obesity-associated cardiovascular risk. Nutrients 2019;11:2664.

36. Karlas T, Petroff D, Sasso M, et al. Individual patient data metaanalysis of controlled attenuation parameter (CAP) technology for assessing steatosis. J Hepatol 2017;66:1022-1030. 


\section{Supplementary material}

Supplementary Table 1 Characteristics of the study population according to weight changes over 1 year

\begin{tabular}{|c|c|c|c|c|c|}
\hline \multirow[t]{2}{*}{ Characteristics } & \multicolumn{5}{|c|}{ Quartiles of weight change } \\
\hline & Quartile 1 & Quartile 2 & Quartile 3 & Quartile 4 & P-value \\
\hline Age (years) & $56.7 \pm 0.7$ & $58.4 \pm 1.0$ & $57.2 \pm 0.6$ & $54.2 \pm 0.7$ & 0.025 \\
\hline Sex (\% men) & $\begin{array}{c}43.7 \\
(37.5-50.1)\end{array}$ & $\begin{array}{c}50.0 \\
(39.3-60.7)\end{array}$ & $\begin{array}{c}49.9 \\
(44.1-55.8)\end{array}$ & $\begin{array}{c}41.0 \\
(35.0-47.4)\end{array}$ & 0.192 \\
\hline Body mass index $\left(\mathrm{kg} / \mathrm{m}^{2}\right)$ & $30.4 \pm 0.5$ & $26.8 \pm 0.6$ & $29.2 \pm 0.4$ & $31.1 \pm 0.4$ & 0.309 \\
\hline Waist circumference $(\mathrm{cm})$ & $103.6 \pm 1.1$ & $96.6 \pm 1.6$ & $100.5 \pm 1.0$ & $104.5 \pm 1.0$ & 0.630 \\
\hline Hypertension (\%) & $\begin{array}{c}48.0 \\
(40.1-56.0)\end{array}$ & $\begin{array}{c}36.6 \\
(25.6-49.2)\end{array}$ & $\begin{array}{c}43.1 \\
(37.0-49.4)\end{array}$ & $\begin{array}{c}44.0 \\
(37.5-50.8)\end{array}$ & 0.377 \\
\hline Diabetes (\%) & $\begin{array}{c}26.0 \\
(21.2-31.4)\end{array}$ & $\begin{array}{c}16.1 \\
(10.7-23.6)\end{array}$ & $\begin{array}{c}17.6 \\
(14.3-21.6)\end{array}$ & $\begin{array}{c}14.4 \\
(10.5-19.4)\end{array}$ & 0.006 \\
\hline $\begin{array}{l}\text { Ethnicity (\%) } \\
\text { Non-Hispanic White }\end{array}$ & $\begin{array}{c}59.9 \\
(52.0-67.4)\end{array}$ & $\begin{array}{c}68.8 \\
(59.7-76.7)\end{array}$ & $\begin{array}{c}71.4 \\
(63.5-78.2)\end{array}$ & $\begin{array}{c}61.4 \\
(53.0-69.2)\end{array}$ & $<0.001$ \\
\hline Non-Hispanic Black & $\begin{array}{c}12.3 \\
(9.0-16.6)\end{array}$ & $\begin{array}{c}8.6 \\
(4.7-15.5)\end{array}$ & $\begin{array}{c}7.6 \\
(5.1-11.2)\end{array}$ & $\begin{array}{c}13.8 \\
(9.5-19.7)\end{array}$ & \\
\hline Hispanic & $\begin{array}{c}16.2 \\
(12.7-20.5)\end{array}$ & $\begin{array}{c}10.9 \\
(6.5-17.7)\end{array}$ & $\begin{array}{c}11.0 \\
(7.7-15.4)\end{array}$ & $\begin{array}{c}15.6 \\
(12.0-20.0)\end{array}$ & \\
\hline Non-Hispanic Asian & $\begin{array}{c}3.3 \\
(2.2-4.8)\end{array}$ & $\begin{array}{c}9.8 \\
(5.9-15.9)\end{array}$ & $\begin{array}{c}7.1 \\
(4.5-10.9)\end{array}$ & $\begin{array}{c}5.1 \\
(3.5-7.4)\end{array}$ & \\
\hline Other & $\begin{array}{c}8.3 \\
(4.7-14.1)\end{array}$ & $\begin{array}{c}1.8 \\
(0.8-4.3)\end{array}$ & $\begin{array}{c}3.0 \\
(2.0-4.4)\end{array}$ & $\begin{array}{c}4.1 \\
(2.7-6.2)\end{array}$ & \\
\hline Smoking (\%) & & & & & 0.036 \\
\hline Never & $\begin{array}{c}51.2 \\
(44.8-57.6)\end{array}$ & $\begin{array}{c}68.0 \\
(57.7-76.5)\end{array}$ & $\begin{array}{c}60.1 \\
(55.2-64.8)\end{array}$ & $\begin{array}{c}57.1 \\
(52.1-61.9)\end{array}$ & \\
\hline Current smoker & $\begin{array}{c}29.8 \\
(25.3-34.9)\end{array}$ & $\begin{array}{c}25.5 \\
(17.8-35.0)\end{array}$ & $\begin{array}{c}27.6 \\
(24.3-31.1)\end{array}$ & $\begin{array}{c}30.2 \\
(24.0-37.2)\end{array}$ & \\
\hline Former smoker & $\begin{array}{c}19.0 \\
(14.7-24.2)\end{array}$ & $\begin{array}{c}6.7 \\
(3.3-13.2)\end{array}$ & $\begin{array}{c}12.4 \\
(9.7-15.7)\end{array}$ & $\begin{array}{c}12.8 \\
(9.2-17.5)\end{array}$ & \\
\hline High education (\%) & $\begin{array}{c}88.1 \\
(84.4-91.0)\end{array}$ & $\begin{array}{c}90.9 \\
(85.8-94.3)\end{array}$ & $\begin{array}{c}89.1 \\
(86.2-91.5)\end{array}$ & $\begin{array}{c}89.3 \\
(85.3-92.3)\end{array}$ & 0.734 \\
\hline Married (\%) & $\begin{array}{c}64.0 \\
(57.2-70.3)\end{array}$ & $\begin{array}{c}69.7 \\
(59.7-78.2)\end{array}$ & $\begin{array}{c}74.0 \\
(71.0-76.9)\end{array}$ & $\begin{array}{c}68.0 \\
(62.5-73.0)\end{array}$ & 0.028 \\
\hline Total cholesterol (mg/dL) & $190.2 \pm 2.8$ & $194.7 \pm 4.3$ & $195.5 \pm 2.0$ & $202.0 \pm 2.5$ & 0.009 \\
\hline $\begin{array}{l}\text { High-density lipoprotein cholesterol } \\
(\mathrm{mg} / \mathrm{dL})\end{array}$ & $53.1 \pm 0.9$ & $55.8 \pm 1.6$ & $54.3 \pm 1.0$ & $53.6 \pm 1.0$ & 0.802 \\
\hline Triglyceride (mg/dL) & $114.8 \pm 4.4$ & $115.5 \pm 14.0$ & $115.9 \pm 6.7$ & $134.9 \pm 9.3$ & 0.078 \\
\hline Fasting glucose (mg/dL) & $114.8 \pm 2.4$ & $111.9 \pm 3.0$ & $115.6 \pm 3.1$ & $109.2 \pm 1.6$ & 0.214 \\
\hline Hemoglobin A1c (\%) & $5.90 \pm 0.05$ & $5.78 \pm 0.08$ & $5.83 \pm 0.04$ & $5.74 \pm 0.02$ & 0.007 \\
\hline Fasting insulin (pmol/L) & $93.3 \pm 10.3$ & $64.2 \pm 7.1$ & $67.0 \pm 3.1$ & $85.5 \pm 6.8$ & 0.343 \\
\hline Alanine aminotransferase (IU/L) & $20.3 \pm 0.5$ & $21.3 \pm 1.0$ & $21.2 \pm 0.6$ & $23.5 \pm 0.5$ & 0.005 \\
\hline Aspartate aminotransferase (IU/L) & $20.5 \pm 0.3$ & $20.8 \pm 0.5$ & $21.1 \pm 0.3$ & $21.9 \pm 0.4$ & 0.019 \\
\hline Gamma glutamyl transferase (IU/L) & $27.7 \pm 1.1$ & $26.0 \pm 2.1$ & $27.7 \pm 1.3$ & $29.9 \pm 1.0$ & 0.149 \\
\hline NAFLD (CAP $\geq 263 \mathrm{~dB} / \mathrm{m}, \%)$ & $\begin{array}{c}55.1 \\
(49.5-60.5)\end{array}$ & $\begin{array}{c}38.6 \\
(28.5-49.7)\end{array}$ & $\begin{array}{c}50.9 \\
(45.0-56.8)\end{array}$ & $\begin{array}{c}65.9 \\
(59.7-71.5)\end{array}$ & $<0.001$ \\
\hline NAFLD (CAP $\geq 285$ dB/m, \%) & $\begin{array}{c}40.9 \\
(33.8-48.4)\end{array}$ & $\begin{array}{c}27.6 \\
(19.4-37.7)\end{array}$ & $\begin{array}{c}37.0 \\
(33.1-41.0)\end{array}$ & $\begin{array}{c}48.5 \\
(41.0-56.0)\end{array}$ & 0.004 \\
\hline Significant fibrosis ( $\geq F 2, \%)$ & $\begin{array}{c}9.7 \\
(6.9-13.4)\end{array}$ & $\begin{array}{c}5.5 \\
(3.0-9.9)\end{array}$ & $\begin{array}{c}7.0 \\
(5.2-9.3)\end{array}$ & $\begin{array}{c}11.6 \\
(8.2-16.1)\end{array}$ & 0.038 \\
\hline
\end{tabular}

Data are shown as the weighted mean \pm standard error or weighted frequency (95\% confidence interval) as appropriate

Triglycerides, fasting glucose, fasting insulin were calculated from fasting subjects only

Range of quartile $1,<-5$ pounds (-2.27 kg); quartile $2,-5$ pounds ( $-2.27 \mathrm{~kg}$ ) to 0 pounds; quartile 3,0 pounds to 4 pounds ( $1.81 \mathrm{~kg}$ ); and quartile $4, \geq 4$ pounds (1.81 kg)

CAP, controlled attenuation parameter; NAFLD, nonalcoholic fatty liver disease 